\title{
Géolinguistique
}

$18 \mid 2018$

Varia

\section{Une étude de la motivation sémantique en domaine albanais : la phytonymie arbëreshe}

A Study of Motivation Semantics in Albanian Domain: Arbëreshe Phytonymia

\section{Maria Luisa Pignoli}

\section{(2) OpenEdition}

12 Journals

Édition électronique

URL : http://journals.openedition.org/geolinguistique/329

DOI : 10.4000/geolinguistique.329

ISSN : 2650-8176

Éditeur

UGA Éditions/Université Grenoble Alpes

\section{Édition imprimée}

ISBN : 978-2-37747-073-0

ISSN : 0761-9081

\section{Référence électronique}

Maria Luisa Pignoli, « Une étude de la motivation sémantique en domaine albanais : la phytonymie arbëreshe », Géolinguistique [En ligne], 18 | 2018, mis en ligne le 01 décembre 2018, consulté le 13 septembre 2019. URL : http://journals.openedition.org/geolinguistique/329 ; DOI : 10.4000/ geolinguistique.329

Ce document a été généré automatiquement le 13 septembre 2019.

Géolinguistique 


\title{
Une étude de la motivation
} sémantique en domaine albanais : la phytonymie arbëreshe

\author{
A Study of Motivation Semantics in Albanian Domain: Arbëreshe Phytonymia
}

\author{
Maria Luisa Pignoli
}

\section{Aspects théoriques : le rôle de la motivation sémantique dans la création lexicale}

1 Les études sur la phytonymie populaire sont encore très peu nombreuses si on les compare à celles sur la zoonymie populaire qui ont contribué à enrichir et à élargir les connaissances géolinguistiques, lexicales et motivationnelles dont l'Atlas Linguarum Europae (ALE) et l'Atlas linguistique roman (ALiR) sont les promoteurs les plus remarquables. Ces recherches considèrent la motivation sémantique comme une composante essentielle du processus de dénomination dans la création lexicale. « Nommer » implique la création d'un nouveau signe qui sert à exprimer et identifier une réalité extralinguistique. Pour que ce processus s'achève il est nécessaire de tirer ses ressources, d'un côté, du système linguistique, notamment des relations morphologiques, lexicales et sémantiques qui existent entre les signes et, de l'autre, du système ethnoculturel qui sous-tend aux représentations que les locuteurs et une communauté linguistique se font de la réalité environnante (Guiraud, 1986 [1967]). Le signe naît motivé, mais une fois introduit dans le système linguistique, il est utilisé par convention, indépendamment de sa motivation ( ibid. : 253) qui tend à s'obscurcir et le signe peut alors sembler arbitraire. Une fois démotivé, le signe peut entrer dans de nouvelles relations au niveau du sens ainsi que de la forme, des relations limitées par la structure du « système lexicogénique » (ibid.: 259) : il peut être remotivé.

Dalbera (2006a) développe davantage le rôle de la remotivation à partir de la considération qu'il a du «motif»: il sert «à classer les objets du monde et à les 
représenter " (ibid. : 21). Bien qu'Alinei $(1996: 12)$ reconnaisse que chaque motivation se traduise par un nom, il insiste sur l'aspect de « nom » de la motivation plutôt que sur celle de représentation et souligne également la relation étroite entre nom et motivation que le nouveau terme icônyme évoque.

Un point essentiel dans l'étude du signe linguistique est la variation dans l'espace que les différentes perspectives de recherche en dialectologie et géolinguistique ont mis en évidence. Alinei considère l'espace linguistique comme centré sur les éléments culturels véhiculés par les désignations, tandis que Dalbera conçoit cet espace comme une projection du temps et il vise plutôt à éclairer le chemin de l'évolution lexicale au niveau tant de la forme que du sens. L'étude des aspects diatopiques et diachroniques des signes permet à Dalbera (2002, 2006a, 2006b) de mettre en valeur la reconstruction sémantique comme étant une composante indispensable de la reconstruction lexicale. En revanche, l'analyse géolexicale intégrée par l'étude motivationnelle permet à Alinei d'aboutir à des résultats novateurs et fondamentaux concernant la reconstruction d'une stratigraphie culturelle dans un espace donné qui met en relation la langue avec l'histoire de la culture. Si du côté anthropologique et ethnographique le lien entre zoonymes de parenté et totémisme est incontestable, Alinei voit la preuve linguistique de l'existence des cultes totémiques se basant sur la vision zoocentrique de l'univers dans l'«importance du côté sémasiologique de la zoonymie populaire " (1997:11) : l'emploi de zoonymes comme iconymes pour désigner des plantes, des maladies, des phénomènes atmosphériques, des êtres magico-religieux et d'autres aspects de la réalité témoigne de cette forme de religion zoocentrique et préhistorique.

4 Tout comme pour les autres niveaux linguistiques, la reconstruction lexicale se base sur la comparaison interne (par confrontation des paradigmes d'un même parler) ou externe (entre plusieurs parlers); pour parvenir à une reconstruction lexicale, il est donc nécessaire d'analyser un corpus "considérable, diversifié, susceptible de permettre la mise en évidence de régularité massives » (Dalbera, 2002: 838). Ces sémantismes sont donc reproductibles et, une fois identifiées les régularités du mécanisme, on pourra reproduire cette démarche pour arriver à saisir la structure la plus profonde du lexique et avoir accès au « sémantisme fondamental » moyennant « la reconstruction des motifs à partir desquels les signes ont été forgés » (Dalbera, 2006a : 36).

Dans cette optique, les résultats de notre étude sur la motivation sémantique en domaine linguistique arbëresh (Pignoli, 2017), une aire albanaise encore très peu étudiée, fournissent de nouveaux matériaux qui pourraient apporter une solide contribution au développement d'une typologie motivationnelle du lexique phytonymique européen. En même temps, cette étude permet d'avancer dans la connaissance du répertoire phytonymique particulier de l'arbëresh d'Italie, qui courait sérieusement le risque de disparaître si on ne l'avait pas recensé.

\section{Aspects géo-historiques : l'Albanie et les flux migratoires historiques}

Les Balkans sont une région qui a toujours été conçue comme "territoire de passage » entre l'Europe occidentale et orientale. La position stratégique du territoire albanais en tant que voie d'accès aux Balkans de la mer Adriatique a toujours été la raison principale qui a poussé les populations étrangères à la conquête de ce territoire. Les conquêtes 
latine, d'abord, et slave et ottomane, ensuite, ont causées une situation d'instabilité politique et, par conséquent, économique et sociale qui a favorisé sans aucun doute le déplacement massif de populations à la recherche de conditions de vie plus stables. C'est dans ce contexte historique et culturel extrêmement fragile que doivent être reconduits les flux migratoires historiques, qui se sont déroulés à plusieurs reprises et dans des directions différentes et qui ont abouti à la formation des établissements arvanites en Grèce (Péloponnèse, îles d'Andros, d'Hydra, d'Eubée, etc.) et de ceux arbëreshë de l'Italie du Sud. Selon les mises à jour historiographiques les plus récentes (Mandalà, 2009), les migrations albanaises vers l'Italie du Sud ont été elles aussi déterminées par plusieurs facteurs, tels que les difficultés économiques et sociales et les conflits militaires dans leur patrie d'origine, en augmentant dans ces populations migrantes le désir de trouver ailleurs des conditions de vie meilleures et plus stables.

\section{L'aire linguistique étudiée : le domaine albanais et ses dialectes}

7 L'albanais est une langue indo-européenne du groupe satem et il est le seul et unique représentant de sa famille linguistique. Il est divisé en deux dialectes principaux: le guègue, que l'on trouve dans le nord de l'Albanie et au Kosovo, et le tosque, dans le sud, en Grèce et en Italie. Les deux aires dialectales sont séparées par la frontière naturelle marquée par le fleuve Shkumbini, qui sépare l'Albanie en deux moitiés.

8 L'albanais est l'une des langues indo-européennes les plus tardivement attestées, tout comme les langues baltes, dont la première attestation écrite n'apparait pas avant le $\mathrm{xVI}^{\mathrm{e}}$ siècle, et le roumain dont le premier texte écrit est daté de 1521. Les premières attestations écrites en albanais remontent au $\mathrm{Xv}^{\mathrm{e}}$ siècle et concernent :

- une « formule de baptême » selon le rite roman et datée de 1462 ;

- la formule de «malédiction de l'Épirote » qui date de 1483 ;

- une petite collecte de 26 mots réunis par Arnold von Harff de Cologne qui est datée de 1496 ;

- une péricope évangélique retrouvée dans un manuscrit grec de la bibliothèque Ambrosienne qui présente la traduction en albanais des vers 62-66 du chapitre XXviI de l'Évangile selon saint Matthieu.

9 En 1555 paraît le Meshari de Gjon Buzuku qui est le premier livre en albanais. Ce manque de témoignages écrits représente l'obstacle le plus considérable à l'étude de l'albanais parce qu'il empêche de reconstituer les phases linguistiques et historiques les plus anciennes de cette langue, ce qui a comme conséquence la persistance de domaines de connaissance flous liés aux origines de la langue albanaise et de sa protolangue, l'illyrien. Une aide importante pour combler en partie le manque de témoignages écrits en ce qui concerne la phase la plus ancienne de la langue albanaise, provient des variétés arbëreshe et arvanites dans lesquelles on peut puiser, car ce sont les variétés les plus archaïques de l'albanais. Malgré l'attestation tardive et le manque de traces concernant la phase linguistique la plus ancienne de l'albanais, les études albanologiques ont contribués - au cours des deux derniers siècles - à mettre en lumière le patrimoine indo-européen et balkanique de la langue albanaise et les traits particuliers de son évolution liés aux périodes préhistoriques et historiques. C'est dans ce contexte que les études de dialectologie albanaise - comme la recherche que nous sommes en train d'illustrer acquièrent une importance vitale strictement liée à l'étude de l'oralité et de sa variation 
spatiale. Donc, les dialectes arbëreshë demeurent - comme toutes les autres variétés dialectales albanaises - les derniers témoins précieux de l'ancien stade de développement de l'albanais, avant 1555.

\subsection{L'Arbëria et les dialectes arbëreshë}

Arbëria est un nom collectif abstrait qui est utilisé pour désigner un territoire dans le sud de l'Italie où de nombreuses communautés albanophones (ou arbëreshe) de la diaspora sont installées depuis plus de six siècles. Il renvoie à l'ancien nom d'Arbër, c'est-à-dire celui de l'Albanie "historique», d'où proviennent ces communautés et arbëresh représente l'ancien nom des Albanais utilisé aujourd'hui pour indiquer les anciennes communautés albanaises de l'Italie et de la Grèce. Les communautés arbëreshe où l'on parle encore des variétés albanaises sont représentées par 50 centres (41 villages et 9 hameaux), distribués dans sept régions de l'Italie du Sud, notamment les Abruzzes, le Molise, la Campanie, les Pouilles, la Basilicate, la Calabre et la Sicile.

11 L'arbëresh, c'est-à-dire l'albanais d'Italie, est une forme d'albanais qui est très influencée par les variétés italo-romanes parlées dans les communautés limitrophes et, surtout, par l'italien régional des aires dans lesquelles on utilise cette variété d'albanais. Cette situation de contact linguistique et de bilinguisme, séculaire et ininterrompu, a déterminé une évolution spécifique de l'arbëresh par rapport aux variétés albanaises parlées dans l'aire balkanique. Les variétés arbëreshe présentent en général une organisation morpho-syntaxique et lexicale qui les rapproche des langues mixtes. Les phénomènes de convergence linguistique opèrent donc avec les phénomènes de divergence qui conduisent à l'introduction d'emprunts lexicaux, avec des modalités spécifiques d'assimilation de ces derniers à la structure morpho-syntaxique de chaque parler arbëresh. Il est en effet possible d'avoir des correspondances lexicales et morphosyntaxiques entre des parlers qui se situent dans des aires parfois même géographiquement éloignées. La conservation d'éléments structuraux et lexicaux, d'une part, et la relexicalisation d'autre part, ont permis à l'arbëresh de se présenter comme une "unité dans la diversité », malgré les différences que l'on trouve entre une variété et l'autre. En tant que type dialectal, l'arbëresh est marqué par une hétéroglossie complexe, puisqu'il constitue le résultat des traits hérités de l'albanais balkanique, ainsi que des traits acquis dans les nouveaux territoires d'installation.

12 En général, le système vocalique arbëresh se présente comme étant beaucoup plus stable et uniforme par rapport à celui des consonnes, avec six voyelles : /i/, /u/, / $/$ /, /ə/, / /, / a/. Les traits caractéristiques les plus importants sont représentés par les différents résultats de [ə] en position tonique et atone, les évolutions des diphtongues originelles * [ie] et *[ua] et la disparition du phonème $/ \mathrm{y} /$ faisant suite à la délabialisation > systématiquement à /i/ (Altimari, 1994 : 240).

13 Par rapport au système consonantique de l'albanais, le consonantisme de ces dialectes montre la présence de la fricative palatale sourde [ç] qui est une spécificité phonétique conservée dans les parlers arbëreshë et qui révèle les emprunts du grec médiéval et moderne. Pour la description des phénomènes de changement phonétique du consonantisme arbëresh nous avons mis à jour la classification par isophones de Solano qui a pris en considération les seuls phonèmes consonantiques intéressés par des processus de changement concernant la latérale alvéolaire vélarisée *[1], les groupes d'occlusive vélaire suivie de latérale *[kl, gl, bl, pl, fl] et la sonorisation de *[x]. Sur la 
base des critères ci-dessus et en utilisant les données récentes, issues des enquêtes de terrain que nous avons menées entre 2007 et 2014, nous avons pu réagencer la distribution diatopique des isophones de Solano en divisant les parlers arbëreshë en six zones principales (Pignoli, 2007), par rapport aux cinq aires que Solano avait indiquées dans son étude : dans la zone $\mathrm{I}, / \mathrm{kl}, \mathrm{gl}, \mathrm{bl}, \mathrm{fl}, \mathrm{pl} /$ sont conservés ; dans la zone II, $/ \mathrm{kl}, \mathrm{gl}, \mathrm{bl}$, $\mathrm{fl}, \mathrm{pl} />$ /c, f, bj, fj, pj/; dans la zone III, /kl, gl, bl, fl, pl/ > /k $\Lambda, \mathrm{g} \Lambda, \mathrm{b} \Lambda, \mathrm{f} \Lambda, \mathrm{p} \Lambda /$; dans la zone IV, $/ \mathrm{kl}, \mathrm{gl} />/ \mathrm{k} \Lambda, \mathrm{g} \Lambda / \sim / \mathrm{kj}, \mathrm{gj} / \sim / \mathrm{c}, \mathrm{J} /$ (voir fig. 1), tandis que /bl, fl, pl/ sont conservés; dans la zone $\mathrm{V}, / \mathrm{x} />\gamma$ et dans la zone $\mathrm{VI}, / \mathrm{l} />/ \gamma / \sim / \mathrm{\text { }} /$ (voir fig. 2).

Figure 1. - Carte de la distribution des isophones dans les zones I, II, III et IV.

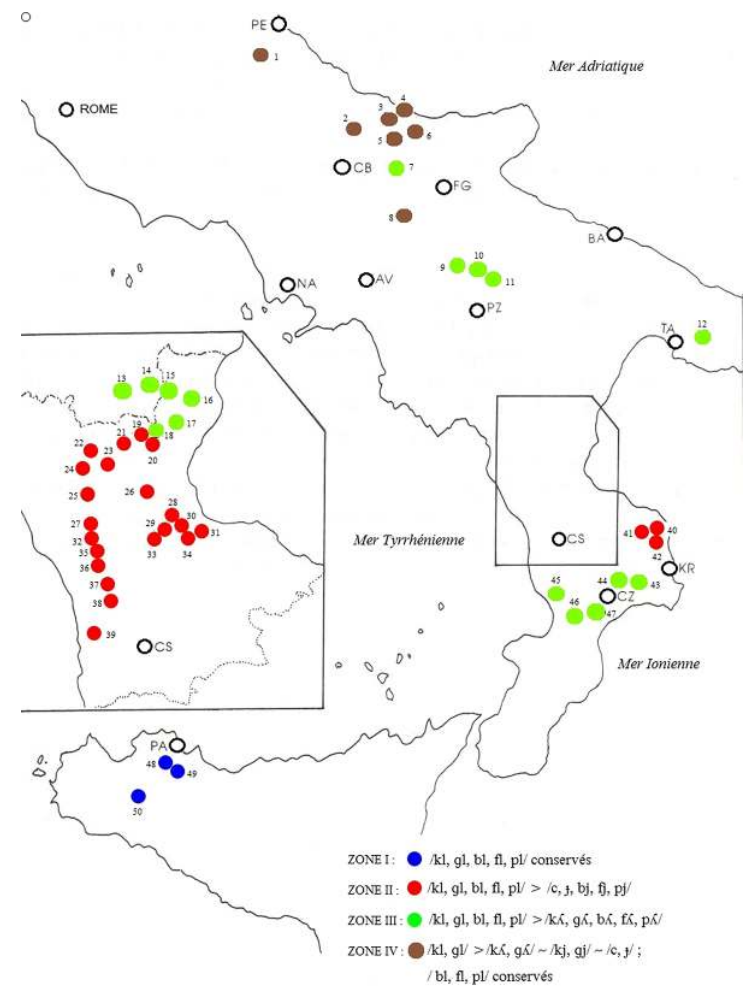


Figure 2. - Carte de la distribution des isophones dans les zones V et VI.

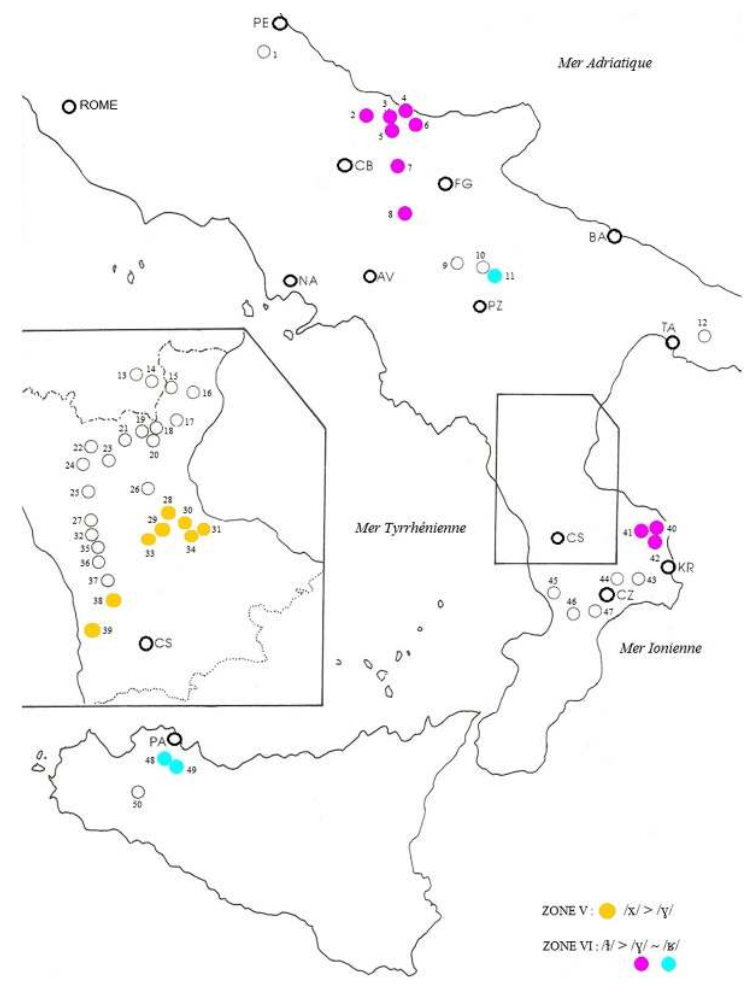

\section{La méthodologie de la recherche}

14 Les phytonymes analysés dans la présente étude ont été recueillis dans huit communautés arbëreshe (voir fig. 3) grâce à des enquêtes de terrain réalisées pendant une période discontinue, comprise entre septembre 2013 et août 2014. Les villages enquêtés ont été Montecilfone et Portocannone, en Molise (respectivement les points numéro 2 et 3 dans la fig. 3 ci-dessous), Greci, en Campanie (point 8), San Costantino Albanese, en Basilicate (point 13), San Giacomo di Cerzeto, Santa Sofia d'Epiro et San Nicola dell'Alto en Calabre (respectivement les points $35,33,42$ ) et Piana degli Albanesi, en Sicile (point 48). 
Figure 3. - Carte des points d'enquête.

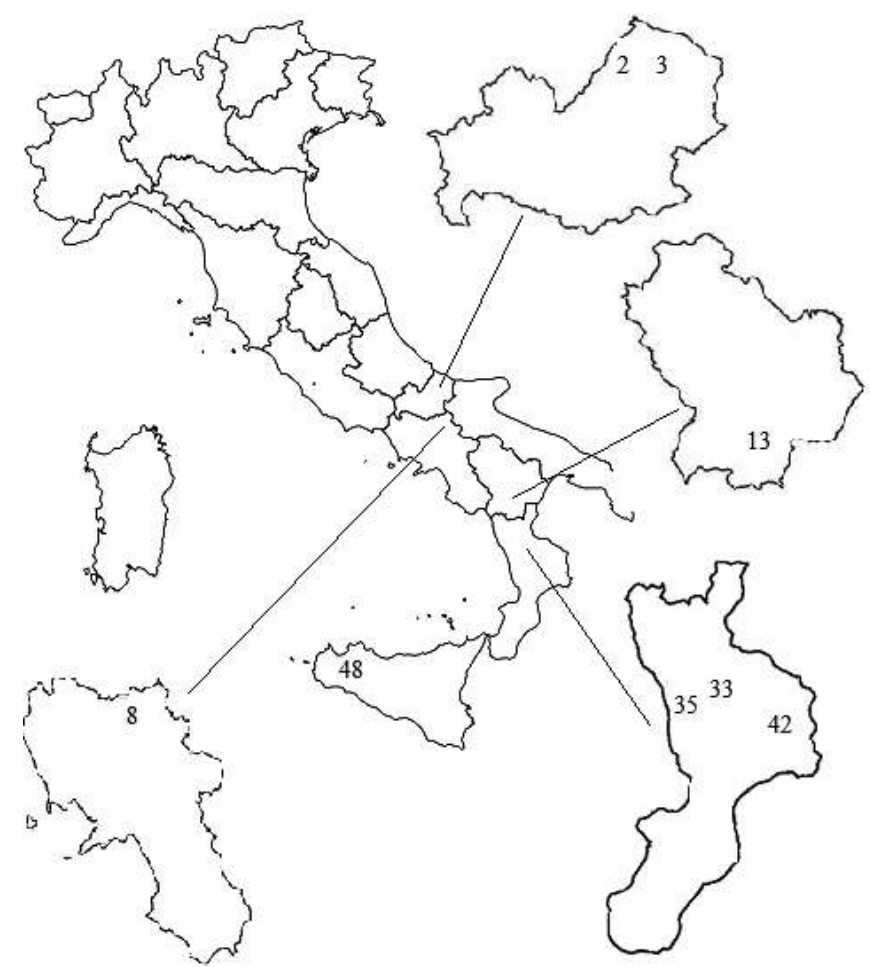

Le questionnaire visuel (voir fig. 4) utilisé nous a permis de faciliter la collecte des données phytonymiques même en dehors des périodes de floraison des espèces végétales, en se servant de "photographies-questions ", compte tenu du sujet particulier de notre recherche et de l'âge des informateurs potentiels qui n'auraient probablement pas pu s'aventurer avec nous dans la campagne ou dans la forêt et se souvenir de toutes les espèces végétales sans une aide concrète, fournie par les photographies des plantes. $\mathrm{Ce}$ questionnaire électronique a été réalisé avec le logiciel FileMaker Pro 13 et il a été structuré selon nos exigences de recherche qui ont pris une forme concrète grâce aux compétences de notre informaticien, Battista Sposato que je remercie infiniment. Le questionnaire contient 2362 "photographies-questions " qui représentent les seules et uniques espèces botaniques florissant dans les régions de l'Italie du Sud où les communautés arbëreshe sont historiquement installées depuis six siècles. 
Figure 4. - Questionnaire visuel, exemple de " photographie-question».

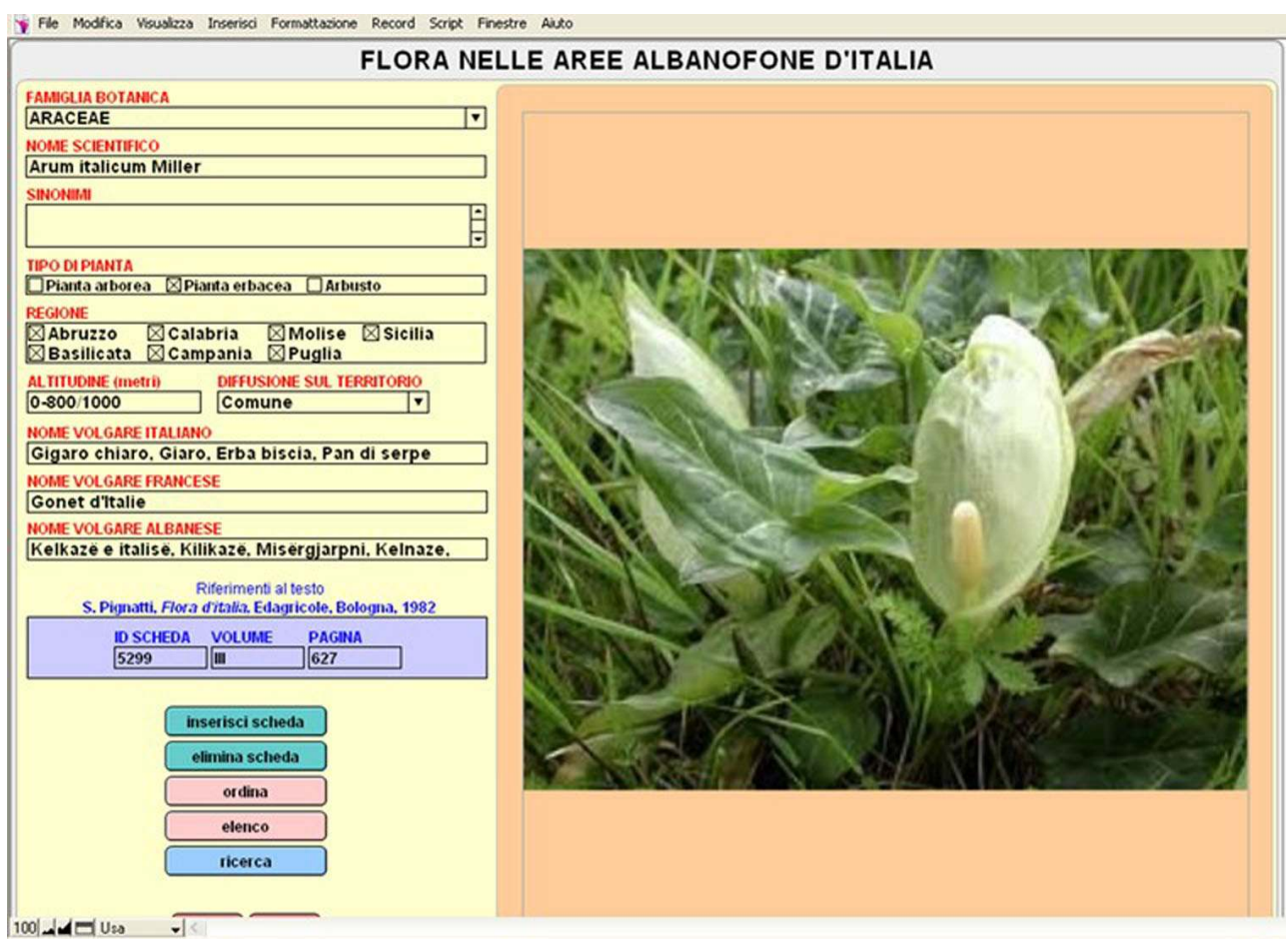

16 L'échantillon de locuteurs interrogés est constitué de 9 hommes et 3 femmes, d'âge supérieur à 70 ans, qui connaissent bien le territoire et l'environnement naturel et qui ont une bonne mémoire ; ils sont tous d'origine arbëreshe, c'est-à-dire qu'ils ont comme langue maternelle un des dialectes arbëreshë et ils ont toujours vécu dans une communauté arbëreshe. L'élicitation des données s'est produite au cours de nombreux entretiens chez les informateurs. Chaque conversation a eu une durée variable - de 60 à 90/100 minutes - strictement liée à la quantité de connaissances botaniques que l'informateur avait et qui lui permettait de reconnaître les espèces photographiées et de nous informer à propos de leur utilisation dans la communauté enquêtée. Tous les entretiens ont été enregistrés avec un magnétophone digital. Le matériel linguistique issu de notre recherche a été stocké et présenté sous forme de base de données, notamment l' Atlas phytonymique arbëresh (APhA) (voir fig.5) qui cherche à fournir un modèle d'exploration et de représentation de ce secteur spécifique du lexique. Grâce encore au support informatique donné à notre projet par Battista Sposato, on a pu réaliser l'APhA qui permet d'ordonnancer et de rechercher les matériaux dialectaux collectés (phytonymes, proverbes, ethnotextes, etc.) de manière plus rapide et précise. 
Figure 5. - L'Atlas phytonymique arbëresh.

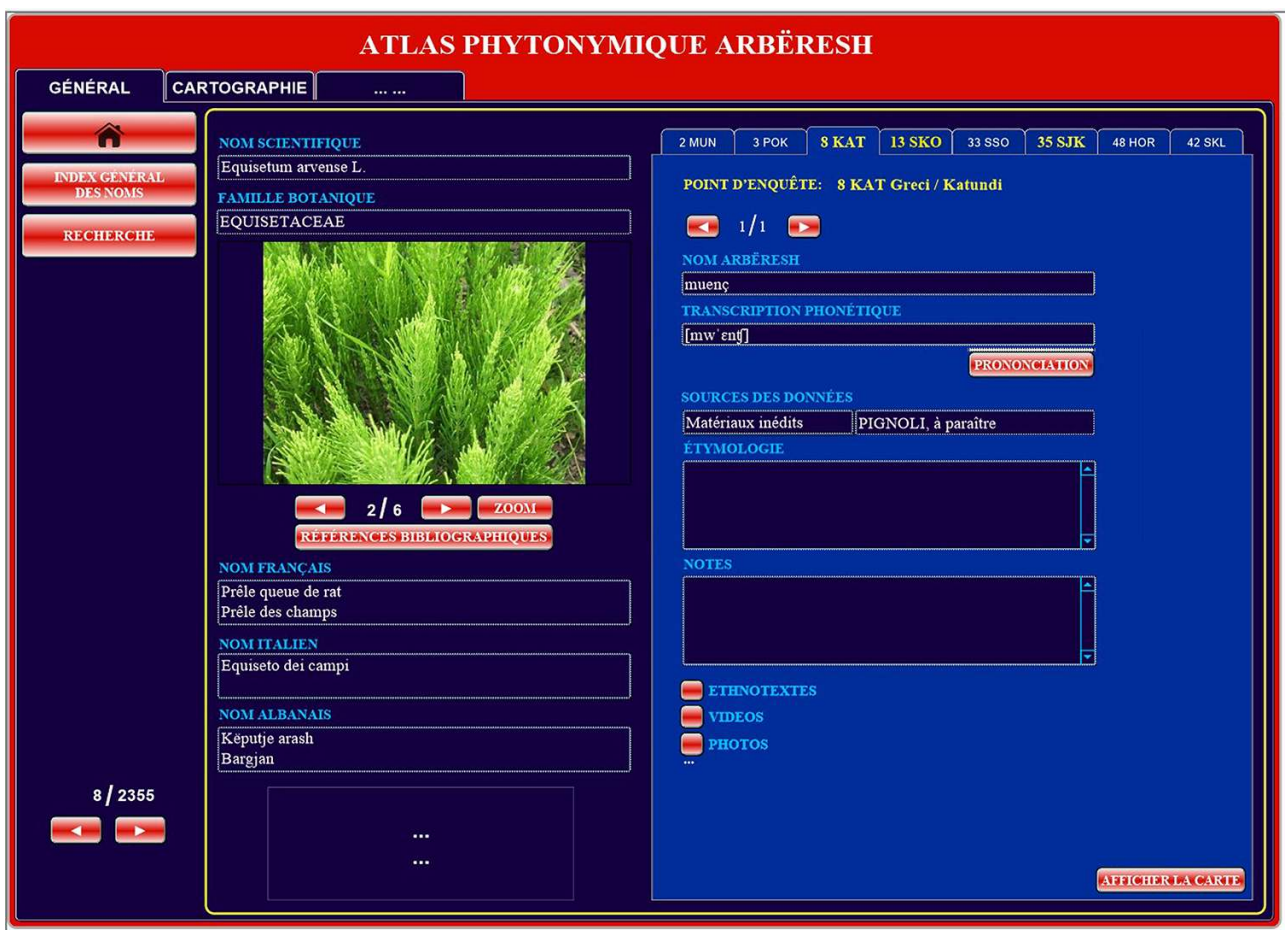

\section{Classement motivationnel des phytonymes arbëreshë relevés}

17 L'analyse des données à notre disposition nous a permis de relever trois classes motivationnelles principales: les phytomorphismes ${ }^{1}$, les anthropomorphismes et les zoomorphismes. N'ayant pas la possibilité d'analyser toutes les désignations rattachées à chaque classe motivationnelle, nous avons choisi d'élaborer les cas les plus significatifs et nous référer brièvement aux autres.

\subsection{Les motivations phytomorphiques}

Les motivations phytomorphiques apparaissent avec une fréquence plus élevée par rapport à toutes les autres classes motivationnelles et, en effet, les phytomorphismes représentent $60 \%$ du corpus motivationnel tout entier. Une présence tellement massive de désignations descriptives dans le corpus phytonymique arbëresh ne doit pas surprendre, car cette typologie motivationnelle appartient à la phase d'évolution onomasiologique plus récente et laisse, par conséquent, des traces plus nombreuses et évidentes dans les systèmes lexicaux des langues. Il s'agit d'une tendance connue en littérature qui a été également mise en évidence dans de nombreuses études sur la zoonymie. Les motivations de ce type sont sans aucun doute le fruit du mouvement constant et «cyclique » qui affecte la motivation et qui amène à « ré-interpréter » et à "re-créer » un signe pour comprendre les mutations que subit le motif à la base d'une unité lexicale à travers des processus de métamorphose qui se succèdent dans le temps et dans l'espace (Dalbera, 2002: 846). Les phytomorphismes comprennent les aspects concernant la morphologie des espèces qui peuvent être perçus à travers les sens et les 
qualités comportementales et d'adaptation des espèces à l'environnement, aux facteurs climatiques et aux manifestations saisonnières de certains phénomènes de la vie végétale, ainsi que les éventuels usages ludiques, alimentaires, thérapeutiques ou domestiques que les espèces peuvent avoir.

\subsubsection{Morphologie de la plante et de ses organes}

Ce premier groupement de motivations descriptives renvoie, moyennant la métaphore, à des images d'objets de différente forme et dimension : [kr'ise $\Lambda]$ « petite croix » et [kr'isje] « croix » renvoient à la morphologie typique de l'inflorescence du chiendent ; [oncz'ar] (< sic. onza) « bouée flottante » renvoie à la forme de la spathe du gouet d'Italie; [kampan 'el ${ }^{\mathrm{h}}$ ] « clochettes » désignent les cyathes de l'euphorbe qui se développent en ombelles terminales; [kart'ut]] (< nap. cartòccio) « cornet de papier, cartouche » dont la forme en cône rappelle la morphologie de la fleur du grand liseron; [cir'ine] « bougies » renvoient aux tiges dressées de la prêle.

Les images relevées dans ce groupement de motivations peuvent aussi renvoyer à la couleur des fleurs ou à l'un des organes de la plante : par exemple [kri'atez] « petite tête dorée » est un nom composé de trois éléments qui se sont agglutinés de la façon suivante : /kri- + -at-+ -ez/ où le nom arb. krye [kr'ie] "tête" a subi l'apocope de sa dernière syllabe /-e/, l'article prépositif/i/, /e/ ou /t/ qui accompagne certains adjectifs, comme l'arb. i, e artë/ t'arta ['a:rt] / ['a:rta] « doré(e) / doré(e)s », tombe régulièrement lorsque l'adjectif intervient dans les processus de formation d'autres noms ou adjectifs et se réduit, dans ce cas, à sa voyelle tonique, tandis que la consonne finale sert à éviter le hiatus qui se crée avec l'ajout du dernier suffixe diminutif -ez; cette forme lexicale décrit, donc, les capitules jaunes et isolés de la centaurée. Les formes lexicales [l'ulja v'Erð] "fleur jaune» et [KuKk'uc], [luluk'uc] et [lelk'uc] «fleur rouge» renvoient respectivement à la couleur des pétales du grand cérinthe et du coquelicot. Les désignations du muscari à toupet, notamment [tfipu $\Lambda^{\prime}$ 'in e k'uce] et [tfipul'in i k'uc] " petit oignon rouge ", renvoient à la couleur des bulbes; en revanche, les formes lexicales [r:S

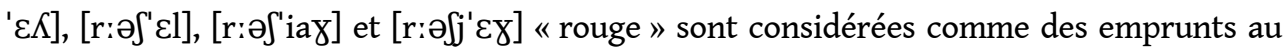

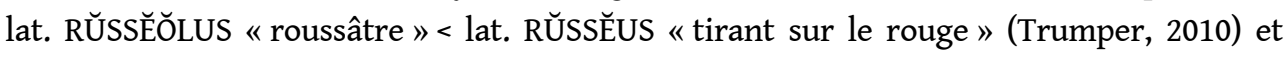
renvoient à la couleur des tiges du laiteron maraîcher.

21 Les images utilisant un terme qualificatif peuvent renvoyer, d'une part, à un aspect de la morphologie du végétal, tel que la rondeur des bulbes du muscari à toupet désigné comme [buKg'az] «enflé, rond» dont la base lexicale bulg- est associée en littérature à l'image de la "rondeur " témoignant ainsi l'origine phonosymbolique de la désignation (Contini, 2009 ; Trumper, 2007) et, d'autre part, à un aspect éthologique de la plante, et

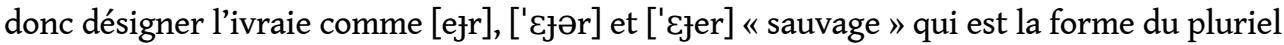
singularisé de l'adjectif alb. [i 'Egər] «sauvage». La forme du pluriel [t'Ełəra] «sauvages » vient de la palatalisation de l'occlusive vélaire sonore du singulier qui est l'une des stratégies de distinction quantitative dont se caractérise la morphologie nominale de l'albanais et ses dialectes.

La direction de la création lexicale va vers la métonymie de la «partie pour le tout » et produit des formes qui renvoient à l'image de l'un des organes de la plante pour en indiquer les propriétés thérapeutiques comme dans le cas de la désignation de l'hellébore [rað'ice] « racine » < lat. RĀDĪX « racine » puisque cette partie de la plante est utilisée en médecine vétérinaire pour la préparation d'une décoction ayant une fonction 
d'antiseptique (Viegi et al., $2003: 233$; Pieroni, 1999: 142) et des propriétés cicatrisantes (Pieroni, 1999: 143; Rexhepi et al., 2013 : 2063). On utilise l'hellébore aussi dans une pratique magique dont Viegi et al. (2003: 240) affirment ne pas savoir quelle est l'origine : la tradition populaire conseille d'accrocher les différentes parties de la plante sur le corps des vaches, brebis et cochons pour empêcher que l'animal tombe malade et pour le traitement de différentes maladies. Il s'agit d'une espèce de talisman fait d'un sachet contenant des petits morceaux de racine de la plante qu'on accroche au corps des animaux (Guarrera, 2006 : 113).

\subsubsection{Onomatopées}

23 Les perceptions auditives permettent la production d'images à base onomatopéique où l'on reproduit le «bruit du piétinement du référent végétal », comme dans le cas de la désignation de la blackstonie perfoliée [tr:iktr:'ak]. Ce phytonyme est analysé formellement comme une séquence de sons « $\mathrm{T}+\mathrm{R}+\mathrm{V}+\mathrm{K}$ » qui peut être rapportée à l'une des structures onomatopéiques identifiées par Guiraud (1986 [1967] : 105) dont le gabarit trilittère TR.K ou sa variante T.K. regroupent les noms désignant les mouvements répétés, saccadés exprimant l'idée de « coup » (Guiraud, 1986 [1967] : 109).

\subsubsection{Transferts phytonymiques}

Dans notre corpus, les transferts "transfèrent " justement les caractéristiques d'une " espèce-image ", c'est-à-dire de la plante qui est prise comme origine pour la création du nom, à l'« espèce-référent » qui fait l'objet de la désignation. Dans la plupart des cas, il s'agit d'images reproduites sur les aspects phytomorphiques communs aux deux espèces, ces aspects ne se basant qu'apparemment et en faible partie sur la morphologie des plantes tout court ; nous estimons en effet qu'il s'agit d'un groupement de noms tabouisés qui reflètent l'importance que ces végétaux ont eu sur le plan magico-thérapeutique, et le transfert concerne donc, avant tout, leurs propriétés intrinsèques et, seulement de façon marginale, leur aspect extérieur commun. Par exemple, l'image "moisissure » à laquelle

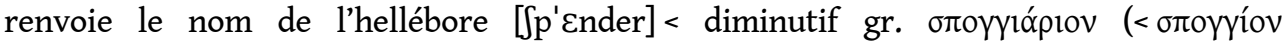
« moisissure, substance toxique » > lat. SPŎNGIIA « champignon ») (Trumper, $2010: 390$ ) donne l'idée de la typologie de transfert tabouistique qui intéresse ces désignations étant donné que l'on n'a pas de traits morphologiques impliqués : les propriétés toxiques de la plante sont représentées par des champignons parasites qui altèrent et corrompent une substance organique, en la rendant dangereuse et toxique; le caractère toxique de l'hellébore est donc considéré à travers la nature toxique même des moisissures.

Le contact avec les organes aériens de certaines espèces d'ortie provoque des troubles cutanés chez l'homme et amène à la création de noms dérivés qui se basent sur l'image de

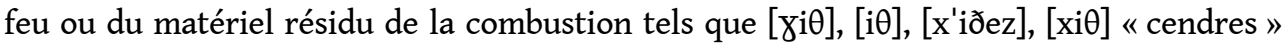
pour désigner l'ortie. Si les propriétés irritantes de l'ortie sont associées à la notion de feu, alors la présence du mot «cendres » nous amène à considérer le fait que le nom du feu peut être tabouisé. Chez les Slaves orientaux, les mots substitutifs de «feu » sont " chaleur ", « tiédeur ", « richesse ", « riche», « clair», « invité », " non ardent» (Zelenin, 1989:176); il est possible que chez les Albanais aussi le feu ait été considéré tabou. À partir de l'arb. /xi:/ «cendres » on ajoute le suffixe /- $\theta$ / pour la formation des diminutifs (Ressuli, $1986: 165)$ ou /-ð/ pour la formation des noms collectifs (Xhuvani \& Çabej, 1962 : 218). 
estimons être basées sur les traits morphologiques communs entre l'« espèce-image » et l'« espèce-référent » et à notre avis, ceux-ci sont en général signalés par la présence, au niveau du signifiant, de formes de suffixation renvoyant à la dichotomie " grand petit " en raison de traits tels que l'aspect, la dimension ou la forme de l'un des organes des deux espèces. Par exemple, l'une des désignations du concombre d'âne [mußun'el] «petit melon » - /mus'un-/ «melon » + /-el/ suffixe diminutif - métaphorise la forme, la taille et l'aspect des fruits; en revanche, la taille et la forme des calices floraux du silène

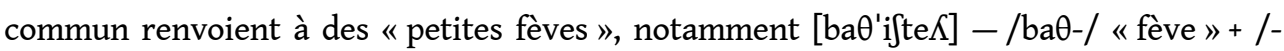
ift-/ suffixe pour la formation de noms collectifs (Xhuvani \& Çabej, 1962: 248) + /-eK/ suffixe diminutif.

Les transferts peuvent être aussi spécifiés par l'adjectif alb. i/e egër "sauvage » dont la valeur sémantique n'est pas toujours la même. Ce spécificateur peut indiquer la "noncomestibilité » d'une espèce par rapport à une autre qui est, généralement, comestible et cultivée, comme dans le cas de [kar'ot sarv'adze] «carotte sauvage » pour désigner la pastanade. Il peut avoir aussi la valeur sémantique de "spontané ", "non cultivé » et indiquer de cette façon la typologie de développement éthologique concernant l'espèce qu'il désigne, qui semble ainsi se distinguer de l'autre espèce similaire mais cultivée, comme dans le cas de l'églantier [trondof i i e e 'Egər] « rose sauvage » et du chardon [kart'’fuł i 'عgər] « artichaut sauvage».

\subsubsection{Habitat de la plante}

Ce groupement de motivations phytomorphiques renvoie, en général, à l'indication du biotope des plantes qui indique, en particulier, l'environnement naturel de croissance des espèces, comme dans le cas des désignations du jonc $[\operatorname{vr\varepsilon } K]$ et $\left[\operatorname{vrul}^{\mathrm{h}}\right]$ « étang, ruisseau » qui suggèrent des bases phonosymboliques de départ, notamment $\beta \mathrm{rVl}$ - / vrVl- qu'on retrouve aussi dans le phytonyme grec à l'origine des désignations arbëreshe ci-dessus, notamment $\beta \rho \circ \tilde{\lambda} \lambda \lambda_{0} v$ "plante poussant dans l'eau » (EDG : 243 ; LG : 98). En particulier, les fricatives labiales en début de mots renvoient à l'impression de souffles mous ou sourds auxquels la séquence de liquide <-r->+ voyelle postérieure donnent un effet de grondement, tel que le grondement d'un torrent ou d'un cours d'eau (Ullmann, 1975 : 107). Cet effet est renforcé par la présence de la liquide latérale qui produit un effet ultérieur de fluidité et qui confirme l'importance de l'élément « eau » dans le processus de désignation de cette espèce végétale. L'image motivante de « eau » est aussi à la base des désignations de la menthe [m'Endər], [m'əndər], [m'əndrez], [m'əndrəz] qui sont considérées comme des emprunts au gr. $\mu$ ív $\theta \eta$ / $\mu$ ív $\theta \alpha$ / $\mu$ ív $\theta$ os dont la source n'est pas sûre à tel point qu'il existe la possibilité qu'il s'agisse d'une langue méditerranéenne (SE, $\mathrm{V}:$ :317), donc d'origine pré-grecque (EDG : 955). Cet hypothèse nous semble tout à fait fondée parce qu'on l'a déjà appliquée aux parcours lexicaux ayant introduit les désignations $\mathrm{du}$ fenouil sauvage et de la mauve - à partir d'une langue méditerranéenne - dans le grec, dans l'albanais et ses dialectes et dans le latin. Ainsi, la racine chamito-sémitique *min- « eau, fleuve » > eg. myn.t « eaux » (HS : 383) > myc. mi-ta

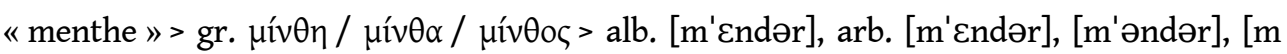
'əndrez], [m'əndrəz], lat. MENTHA, MENTA. 


\subsubsection{Emploi de la plante} espèces ayant de puissantes propriétés médicinales (toxique ou bénéfiques) très connues et exploitées en médecine populaire et en médecine vétérinaire, telles que les propriétés du gouet d'Italie nommé [r: 'ufi f'arprit] « raisin du serpent », du muscari à toupet nommé [cepd'osje] « oignon de truie », du silène commun nommé [b'aOza 'ußkut] « petite fève du loup » et de la morelle noire nommée [pumð' Jri f' arprit] « tomate du serpent ».

31 L'image "nourriture " peut être aussi spécifiée par le nom d'un personnage religieux et désigner, de même que le zoonyme, les propriétés médicinales d'une espèce comme dans le cas de la grande brize qui est nommée [b'uka $\varepsilon$ bumbin'el:it] « pain de l'Enfant Jésus ».

L'emploi thérapeutique de la prêle des champs est véhiculé par l'image "casse-mets » [stokam' $\varepsilon n t]$ < cal. stocca e mmenta (NDDC : 689) qui désigne cette espèce et qui renvoie aux propriétés vulnéraires des tiges de la plante que l'on casse justement et que l'on applique directement sur les blessures : la désignation représente une sorte de «mode d'emploi » de la plante.

\subsection{Motivations anthropomorphiques}

Le deuxième groupement typologique majeur que l'analyse de nos données a mis en évidence est celui des anthropomorphismes, qui regroupe les désignations renvoyant aux noms de parenté, de personnages religieux païens ou chrétiens, aux activités humaines et aux noms des parties du corps humain. Les anthropomorphismes représentent un pourcentage beaucoup plus faible par rapport à celui des phytomorphismes: ils correspondent à $16 \%$ des motivations identifiées. Ils représentent le stade onomasiologique moyen dans la stratigraphie envisagée par Alinei (1984) et confirment donc le schéma évolutif tracé par cet auteur selon lequel, avec le passage de la société primitive à une organisation sociale basée sur la distinction entre les classes, l'« animalparent" se traduit, tout d'abord, dans un être anthropomorphique païen (esprits, sorcières, elfes, lutins, etc.) et puis, dans un être anthropomorphique appartenant à une religion monothéiste, chrétienne ou islamique (saints, démons, madones, Allah, etc.) (Alinei, 1984 ; Caprini, 1998) ayant tous les pouvoirs « magico-religieux » distribués, dans la phase évolutive primitive, entre les différentes catégories du réel à travers lesquelles l'« animal-parent » se manifestait (Alinei, $1984:$ 75). 


\subsubsection{Noms de parenté}

34 « ancêtres » renvoie à la violette odorante et celle "petite mère » renvoie à l'amarante. Les noms qui désignent la violette odorante [monos'ace], [manus'ace] et [manust'ace] sont d'héritage oriental (Dizdari, 2005 : 616) et peuvent être rapprochés d'un ensemble de noms désignant la violette odorante ou d'autres espèces du genre Viola ssp.: alb. manushaqe, tu. menekşé, arm. manyeshág, bulg. МУМУШАНКА (mumushánka), ar. banafsaj. Une correspondance ultérieure se trouve reflétée dans un mot dérivé skr. manushyá qui a le sens de " humain, viril, utile ou bon pour l'homme », " un homme, un être humain », et signifie, dans un sens collectif, « un groupe d'ancêtres décédés » (SKRED : 784). L'analyse morphologique de ces noms permet de remarquer la présence de $\left[-\int-\right]<-$ sh- $>$ ou $[-$-s- $]<-$ s- >, <-ş- > qui en albanais introduit un ablatif pluriel avec valeur de collectif (Xhuvani \& Çabej, 1962:272) et en sanskrit -shyá a la fonction de spécificateur de quantité dans les noms composés (SKRED : 1108) qui fait donc acquérir au terme un sens collectif; la confirmation du sens collectif de ces noms nous arrive de l'arabe où la dénomination de la violette banafsaj est un nom collectif (DMWA:94). En effet, cette espèce botanique à rhizome rampant produit de nombreux stolons florifères aériens et, poussant ainsi horizontalement, se répand sur des aires étendues avec ses fleurs typiques de couleur violette qui forment avec les feuilles un véritable gazon uniforme qui donne l'image d'un «ensemble de violettes». Du côté des croyances populaires, Beccaria (1995:221) affirme que depuis un temps immémorial, les hommes ont doté les herbes d'une âme végétale et qu'il s'agit normalement d'herbes très communes, à l'apparence insignifiantes, mais avec une odeur intense, âcre: les odeurs fortes, agréables ou irritantes font fuir, selon la croyance populaire, les diables et les sorcières.

La croyance d'origine animiste que Donini (1964: 69) définit, dans la «théorie du mana », comme un pouvoir mystérieux enveloppant les hommes et résidant dans les objets, les animaux et les plantes, a amené à renforcer notre hypothèse de dérivation des noms arbëreshë de la violette du sanskrit; en outre, dans cette langue, on peut rencontrer le phytonyme skr. mana désignant le nard indien - (Nardostachys jatamansi L.) une plante à forte odeur - et le fils du démon Śambara (SKRED : 783). Dans le récit mythologique d'Attis et Cybèle, la violette est une fleur d'amour qui incarne l'esprit du dieu-amant Attis, en le diffusant par l'intermédiaire de son parfum intense (Frazer, 1973). Si l'idée de sacré que cette espèce a représentée est patente en raison de son odeur forte qui était interprétée comme la présence divine de l'esprit d'un ancêtre décédé, il ne faut pas oublier les nombreuses propriétés magico-thérapeutiques de la plante, notamment laxatives, émétiques, diurétiques, sédatives, vulnéraires (Guarrera, 2006) et ses fonctions propitiatoires et bénéfiques qui font fuir les mauvais esprits et rendent l'année agricole propice (Atzei, $2003:$ 476).

L'image "petite mère» désigne l'amarante réfléchie qui est nommée [n'Enez]; ce phytonyme se compose d'une base lexicale polysémique /nعn-/ + le suffixe diminutif féminin /-ez/. Selon l'opinion de Trumper (2010), la base lexicale de départ /nVn-/ est caractérisée par l'ancienne alternance IE. de la voyelle thématique : /-a-/ /-e-/ /-ə-/ /-i-/ /-o-/ /-u-/ s'alternent en fait dans plusieurs noms, tels que : l'arb. [nan] « grandmère ", « vieille »; l'alb. [nan], [nən] et [n'əne] "grand-mère »; l'alb. [nєn] et [n'єnəz] « mère "; l'arb. [non] "grand-mère, grand-père »; l'arb. [nun] «parrain/marraine, témoin de mariage »; l'arb. [n'Enez] «larve de mouche-bleue» et [n'ənəz] «larve de 
mouche ». Le phytonyme arbëresh trouve aussi d'autres correspondances : en aroum. nenă, nană « amarante, sanicle d'Europe »; en gr.m. vévo « différentes espèces d'amarante »; en bulg. nané «menthe»; en tu. naná «menthe»; en irl.m. nenaid, gal. dynad (<*nynad), bret. linad, avec dissimilation, angl. nettle, all. Nessel, signifiant tous « ortie » (Trumper, 2010 : 382). À bien regarder, tous ces noms renvoient à une seule et unique motivation parentélaire : mère signifiant "nourrice », c'est-à-dire qui donne la nourriture; de cette interprétation sont issus les noms de l'amarante, de la menthe et de l'ortie, herbes très nourrissantes et dotées aussi de propriétés phytothérapeutiques indiscutables; tout comme ces espèces botaniques, les larves ont représenté pour des millénaires un élément de base de l'alimentation humain (Alinei, 1984 : 77).

\subsubsection{Anthroponymes}

Les données de notre corpus témoignent la présence d'un seul anthroponyme désignant le gouet d'Italie: [məygandr' $\varepsilon$ :] est une désignation composée de deux noms propres, notamment les diminutifs hypocoristiques [məyg-] (<Dominique) et [-ndrع:] (< André). Les femmes de la communauté de Greci nous ont parlé des propriétés presque miraculeuses des feuilles de cette espèce dont la sécrétion sortant des feuilles soigne les blessures et les plaies de la peau, les verrues, les engelures, les furoncles, les hémorroïdes, les arthrites, les contusions et les rhumatismes (Guarrera, 2006:60). En revanche, les croyances populaires donnent au gouet des pouvoirs magiques - permettant de faire des pronostics sur les récoltes et les productions agricoles - et qui sont témoignés, par exemple, par les dénominations que l'espèce a en Basilicate et en Ligurie, respectivement bona annata et buna anná « année bonne » (Penzig, 1924: 53; Beccaria, 1995 : 220). Il est intéressant de noter que les deux anthroponymes utilisés pour nommer le gouet renvoient à deux saints - saint Dominique et saint André - qui font l'objet de deux cultes différents dans les traditions populaires du sud de l'Italie. Dans la communauté abruzzaise de Cocullo, saint Dominique protège et soigne les hommes des morsures de serpent et ce culte chrétien remonte au culte païen de la déesse serpent Angita, ancienne divinité de la Marsica ayant le pouvoir d'enchanter les serpents et de soigner les blessures qu'ils causent (Lombardi Satriani, 1971:97). Dans la communauté d'Amalfi, saint André est le patron des marins et de son corps sort une manne miraculeuse que les fidèles ont l'habitude de prendre en trempant des petits tampons d'ouate à utiliser, avec la plus grande confiance, en cas de maladie ou de calamité (Lombardi Satriani, 1971 : 205). Il est incontestable que le gouet d'Italie, en particulier ses propriétés thérapeutiques soignant la peau, sont reconnues et vénérées par les hommes, et sur le plan linguistique cette vénération et reconnaissance sont traduites moyennant l'utilisation d'anthroponymes qui remplacent les termes d'origine. On peut supposer que l'utilisation de ces deux anthroponymes marque, d'une part, le respect que l'on montre à la plante et, d'autre part, la reconnaissance de ses vertus thérapeutiques et miraculeuses. La fonction des diminutifs hypocoristiques marquerait davantage l'affection qu'on a voulu montrer à la plante.

\subsubsection{Noms d'êtres magico-religieux païens et chrétiens}

Un développement sémantique ultérieur des anthropomorphismes est représenté par les désignations de l'orge maritime et de la prêle des champs. L'image "lutin sauvage "

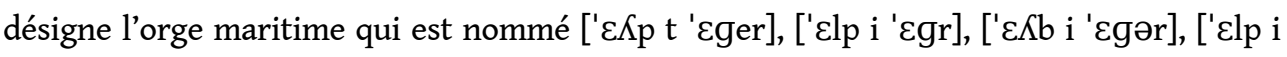




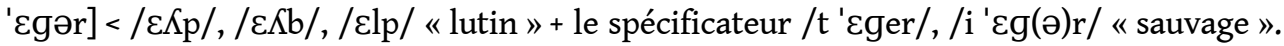
Les formes $/ \varepsilon \Lambda \mathrm{p} /, / \varepsilon \hat{\mathrm{b}} / \mathrm{l}, / \varepsilon \mathrm{lp} /$ peuvent être rapprochées des formes qu'on trouve en all. Alp «cauchemar», all. Elfen «lutin» et en v.h.a. Alb «sauterelle»; ce dernier nom indiquait d'abord les lutins mythiques et ensuite le cauchemar, comme dans les noms de quelques herbes magiques utilisées contre le cauchemar: all.dial. Alfkrok «herbe du cauchemar» pour le millepertuis, Alpranken «vrille du cauchemar» pour l'espèce Solanum dulcamara L., Alfkraut " herbe du cauchemar » pour l'espèce Solanum nigrum $\mathrm{L}$. (Beccaria, $1995: 203$ ). La polysémie des formes allemandes Alb, Alp, Elfen peut être comparée aux bases lexicales albanaises alb-, elb, elp- qui sont utilisées pour la formation des noms Bardha «figures mythologiques blanchâtres de brouillard» (Lambertz, 1973: 466) (< $i$ bardh «blanc» < *baldh < alb-), elb / elp « orge » et pelë « jument» (< elp avec métathèse). La présence du cheval comme animal en relation avec les esprits est présent dans la mythologie populaire germanique où cet équidé et la jument sont les manifestations préférées de la Mara, c'est-à-dire du cauchemar (Caprini, 1984). Les populations de la Mélanésie et de la Polynésie considèrent, aujourd'hui encore, le blanc comme symbole de mort parce qu'il est associé à la couleur blanche des revenants (Lanternari, 1959:99) et dans la littérature orale balkanique le cheval blanc est l'épiphanie des âmes des morts (voir la Ballade de Costantin et Garentine). L'espèce botanique est classée parmi les mauvaises herbes et elle est considérée comme l'une des espèces les plus envahissantes, tandis que les croyances populaires donnent à la plante des pouvoirs magiques (Guarrera, 2006: 304; Atzei, 2003 : 178). Il est possible que sur le plan linguistique, l'anthropomorphisme païen désignant cette plante traduit, d'un côté, la dimension mythique et magique des croyances populaires et, de l'autre, les effets dévastateurs que la plante a sur les terrains où elle pousse; en revanche, l'autre désignation de la plante ['a:ra dj'alӨit] «champ du diable » témoigne de l'évolution motivationnelle vers le stade anthropomorphique chrétien et plus récent qui est mis en évidence par la présence du diable dans le syntagme dénominatif.

La désignation de la prêle des champs [mw'Ent]] «moines » qu'on utilise à Greci est un emprunt du nap. muónece « moines» (NVDN : 432). L'image motivante « moines » traduit sans doute les nombreuses qualités thérapeutiques dont la plante est dotée et qui sont bien représentées par les moines médiévaux, c'est-à-dire les détenteurs de la tradition et de la pratique de la médecine populaire.

\subsubsection{Activités humaines}

Ce groupe de désignations est représenté par des termes liés aux activités humaines, comme l'atteste, par exemple, les noms du cabaret des oiseaux [karða $\left.\Lambda^{\prime} \varepsilon f\right]$ " cardeur » et

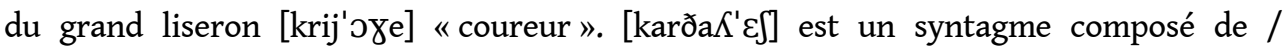
karða-/ < cal. scardare " carder » (NDDC : 618) + le mot arb. /-KES/ « laine » et cette image renvoie à l'utilisation que les cardeurs faisaient des têtes florales de l'espèce, lesquelles allaient former les peignes de la carde une fois séchées. [krij'oye] «coureur » est un emprunt du nap. curriúlo « liseron » (< currià « courir, s'enfuir ») (NVDN : 244) et il renvoie à l'idée du port grimpant de la plante qui se déploie (qui « court») vers le haut. Il s'agit donc de désignations liées, d'une part, à l'utilisation de la plante ou de l'une de ses parties dans la vie quotidienne et, d'autre part, à la description d'une caractéristique éthologique ou morphologique du végétal moyennant une métaphore. 


\subsubsection{Parties du corps humain}

41 Les motivations basées sur une partie du corps humain, auxquelles s'ajoutent quelques spécificateurs (/i 'Eger/ ou /sarv'atf/ « sauvage »), désignent généralement des végétaux ayant des propriétés thérapeutiques; en revanche, un petit pourcentage de ces désignations se réfère aux caractéristiques phytomorphiques des plantes. Ainsi, les images «menton» et «barbe» renvoient respectivement aux puissantes propriétés

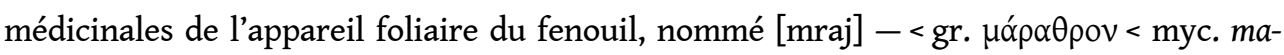
ra-tu-wo < eg. mrt «menton » en raison des rapports commerciaux entre Mycéniens et Égyptiens (HS : 390) - [mraj t 'Eger], [mraj e 'Egər], [mra sarv'at5] et de la prêle des champs, nommée [mj'Ekər], puisque la barbe et, par conséquent, la partie du corps où elle pousse sont le siège de la puissance (Beccaria, 1995 : 219). La désignation de la picride à Santa Sofia d'Epiro est [jaftaj'ifte $\mathcal{C}$ 《 six petit doigts » et renvoie à la forme et l'aspect des feuilles de la plante qui donnent au végétal une forme à " plusieurs feuilles divisées ", comme les doigts des mains.

\subsection{Motivations zoomorphiques}

Le troisième groupement important de motivations tiré de notre corpus concerne les zoomorphismes, qui sont ces désignations où la plante est représentée par un animal: elles correspondent à $13 \%$ des motivations identifiées puisque cette typologie motivationnelle, tout comme celle des motivations parentélaires, représente le stade onomasiologique le plus ancien et, par conséquent, le moins attesté par rapport aux anthropomorphismes et aux phytomorphismes.

\subsubsection{Zoonymes}

Les données de notre corpus montrent que les représentations zoomorphiques renvoient toutes aux animaux totémiques sacrés tels que cerf, bœuf, loup, renard, serpent, cheval/ jument, milan, chien, etc., qui traduisent, sur le plan linguistique, les propriétés magicothérapeutiques dont les espèces sont dotées. Ainsi, les images "petite jument", « sauterelle » et " petit bœuf » désignent, respectivement, la prêle des champs nommée [peKik'om] (</pe $\Lambda$-/ « jument » + suffixe diminutif /-ik-/ + suffixe pour la formation des

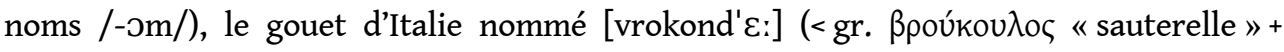
suffixe alb. - e pour la formation de noms dénominaux) et le grand roseau nommé [kaym], [k'ałmer], [kałm], [k'amus] (</ka-/ «bœuf»+ suffixe diminutif /---/, /-ł-/, /-- - / + suffixe pour la formation du pluriel /-m/) et renvoient aux puissantes propriétés magicothérapeutiques dont ces espèces sont dotées. Il est possible de rencontrer aussi toute une série d'oiseaux : l'image " petit milan » désigne la carotte sauvage nommée [c'ifter] $(</ c$ 'ift-/ «milan »+ suffixe diminutif /-er/) et renvoie aux vertus thérapeutiques du végétal ; en revanche, l'image « cygne » désigne la morelle noire nommée [ $\Lambda \mathrm{ab}$ 'Jt] et cette forme lexicale correspond aux noms slaves du cygne slo. labod, slvc. labut, tch. labut, bos. labud, etc. (EWSS : 162). Les croyances populaires sur le territoire euro-asiatique associent la couleur blanche aux revenants et les oiseaux aquatiques aux messagers de la mort (Riegler, 1981: 309) ; l'utilisation de l'image " cygne » pour désigner ce végétal est donc l'expression du danger représenté par la plante en raison de ses propriétés toxiques. 


\subsubsection{Parties du corps animal}

44 La structure motivationnelle "partie du corps animal + animal» est l'une des plus communes dans notre corpus et, en général, en phytonymie. Elle est utilisée pour les désignations où une partie de la plante représente tout le végétal. En effet, il s'agit de métonymies de «la partie pour le tout » où la partie du corps animal représentant la motivation principale symbolise la plante elle-même, tandis que le zoonyme métaphorise la vertu thérapeutique ou les propriétés nuisibles du végétal. Ainsi, dans notre corpus les images "oreille de lièvre ", "langue de vache » et "queue de renard» renvoient sans doute aux appareils foliaires du gouet d'Italie nommé [veflj'Epuri] (/vef-/ « oreille » + /-lj 'Epuri/ « de lièvre »), de la buglosse azurée nommée [f'uxa lops] (/f'uxa/ «langue »+ / lops/ « de vache ») et de la prêle des champs nommée [bift ð'Elpər] (/bift/ « queue »+/ð 'Elpər/ «de renard/) respectivement, et les zoonymes spécifient qu'il s'agit de plantes connues pour leurs propriétés médicinales. En revanche, les aspects métaphorisés dans les images "moustache de chat» et «boyau de poule» désignant, respectivement, l'égylope ovale nommée [must'єca m'atfas] (/must' $\varepsilon c a /$ «moustache»+ /m'atfas/ « de chat ») et le liseron nommé [z'or:a puls] (/z'or:a/ «boyau»+/puls/ « de poule ») sont négatifs puisque les spécificateurs zoomorphiques choisis pour représenter les propriétés mauvaises de ces espèces envahissantes sont considérés, par les anciennes croyances populaires, comme les épiphanies du diable (Riegler, 1981: 317).

La valeur sémantique d'un spécificateur zoomorphique est clairement liée au concept totémique dualiste selon lequel l'animal peut être protecteur et ennemi de l'homme si l'on ne respecte pas certains tabous : cette ambiguïté de base est le reflet du lien entre totem et tabou, entre sentiments du sacré et terreur (Alinei, 1984:81) qui réglaient les relations entre les hommes et les animaux totémiques vénérés et, en même temps, craints. Le zoonyme en position de spécificateur explicite de manière remarquable cette ambiguïté des relations, en renvoyant aussi bien aux qualités positives et bénéfiques de la plante, qu'aux qualités négatives et nuisibles, tout comme lorsqu'il se trouve seul pour désigner les êtres végétaux. Nous croyons donc que le choix de l'animal est tout à fait arbitraire dans ce type de structures parce que les "animaux-spécificateurs » ont des valeurs sémantiques interchangeables entre eux et ils renvoient uniquement au «caractère sacré » des plantes; le choix du zoonyme est donc arbitraire parce qu'il dépend - comme l'affirme Alinei (1984) - de pertinences culturelles qui conduisent à rapprocher une espèce végétale d'une autre animale. En effet, dans la phytonymie arbëreshe on n'a pas retrouvé la présence de l'ours, bien qu'il soit l'un des animaux totémiques les plus importants et les plus présents dans les ethnoclassifications; d'autres espèces totémiques sont également absentes telles que le crapaud et le coucou.

\section{Conclusion}

L'analyse motivationnelle des phytonymes arbëreshë a permis, d'abord, de confirmer le rôle important que la motivation a dans le processus de dénomination et, ensuite, elle a donné de nouvelles possibilités d'interprétation des nombreux phytonymes albanais caractérisés par un étymon inconnu ou incertain. D’ailleurs, créer un nouveau signe signifie mettre en relation les ressources d'un système linguistique avec celles d'un système ethnoculturel de représentation de la réalité environnante que les locuteurs et les communautés linguistiques adoptent (Guiraud, 1986 [1967]) et, par conséquent, 
l'exploration de la dimension culturelle doit toujours être prise en considération lors des analyses linguistiques, comme nous avons essayé de le faire avec cette étude. Comme nous l'avons mis en évidence, par ailleurs, la motivation est le résultat de perspectives pluridimensionnelles qui ne sont pas limitées à la simple description des caractéristiques des référents visibles à l'œil nu et, surtout dans le cas des désignations zoomorphiques et anthropomorphiques, le recours aux aspects ethnoculturels nous a permis de rendre la lecture de la motivation plus transparente.

\section{BIBLIOGRAPHIE}

AlineI Mario, 1984, Dal totemismo al cristianesimo popolare. Sviluppi semantici nei dialetti italiani ed europei, Alessandria, Edizioni dell'Orso.

Alinei Mario, 1996, « Aspetti teorici della motivazione », Quaderni di Semantica, vol. 17, $\mathrm{n}^{\circ} 1$, p. 1-17.

ALINEI Mario, 1997, « Magico-Religious Motivations in European Dialects: A Contribution to Archeolinguistics », Dialectologia et Geolinguistica, n 5, p. 3-30.

AltimARI Francesco, 1994, « La parlata di Macchia Albanese: appunti fonologici », dans F. Altimari et L. Savoia (éds), I dialetti italo-albanesi, Rome, Bulzoni Editore, p. 239-258.

ATZEI Aldo Domenico, 2003, Le piante nella tradizione popolare dela Sardegna, Sassari, Carlo Delfino Editore.

BECCARIA Gian Luigi, 1995, I nomi del mondo. Santi, demoni, folletti e le parole perdute, Turin, Einaudi.

CAPRINI Rita, 1984, « Nighmare », Quaderni di Semantica, vol. 5, nº 1, p. 17-33.

CAPRINI Rita, 1998, « Animali totemici: l'esperienza di 'Quaderni di Semantica' », L'immagine riflessa, vol. 20, $\mathrm{n}^{\circ} 2$, p. 221-236.

CONTINI Michel, 2009, «Les phonosymbolismes : continuité d'une motivation primaire?», Travaux de linguistique, vol. 2, $\mathrm{n}^{\circ}$ 59, p. 77-103. Disponible sur <www.cairn.info/revue-travaux-delinguistique-2009-2-page-77.htm> [dernier accès le 25 août 2018].

DALBERA Jean-Philippe, 2002, « Géolinguistique : un nouveau souffle? », Revue belge de philologie et d'histoire, $\mathrm{n}^{\circ} 80$, p. 831-849.

DALBERA Jean-Philippe, 2006a, Des dialectes au langage, Paris, Honoré Champion.

DALBERA Jean-Philippe, 2006b, « Zoonymes et relations parentélaires : réflexions sur la belette. De l'étymologie à la reconstruction lexicale ", Quaderni di Semantica, vol. 27, n 1-2, p. 225-252.

DAM = GIAMMARCo Ernesto, 1976, Dizionario abbruzzese e molisano, vol. 3, Rome, Edizioni dell'Ateneo.

DIZDARI Tahir, 2005, Fjalor i orientalizmave në gjuhën shqipe, Tirana, AIITC.

DMWA = WEHR Hans, 1979, A Dictionary of Modern Written Arabic, Urbana, Spoken Language Service, INC. 
Donini Ambrogio, 1964, Lineamenti di storia delle religioni, Rome, Editori Riuniti.

EDG = BEEKES Robert \& VAN BEEK Lucien, 2010, Etymological Dictionary of Greek, Leiden-Boston, Éditions Brill.

EWSS = MIKLOSICH Franz, 1886, Etymologisches Wörterbuch der Slavischen Sprachen.

FRAZER James, 1973, Il ramo d'oro, Turin, Editore Boringhieri.

GUARRERA Paolo Maria, 2006, Usi e tradizioni della flora italiana. Medicina popolare ed etnobotanica, Rome, Aracne.

GUIRAUD Pierre, 1986, Structures étymologiques du lexique français [1967], Paris, Larousse.

HS = OREL Vladimir \& STOLBOVA Olga, 1995, Hamito-Semitic Etymological Dictionary, Berne-Munich, Francke Verlag.

LAMBERTZ Maximilian, 1973, « Die Mythologie des Albaner », dans H. W. Haussig (éd.), Wörterbuch der Mythologie, vol. 2 : Götter un Mythen im Alten Europa, Stuttgart, Ernst Klett, p. 455-509.

LANTERNARI Vittorio, 1959, La grande festa, Bari, Dedalo.

LG = RoHLFs Gerard, 1964, Lexicon Graecanicum Italiae Inferioris, Tübingen, Max Niemeyer.

LOMBARDI SATRIANI Luigi, 1971, Santi, streghe e diavoli, Florence, Sansoni Editore.

MANDALÀ Matteo, 2009, Mundus vult decipi, Rende, Fondazione « Solano ».

NDDC $=$ RoHLFS Gerard, 2010, Nuovo dizionario dialettale della Calabria, Ravenne, Longo Editore

NVDN = D'Ascoli Francesco, 1993, Nuovo vocabolario dialettale napoletano, Naples, Gallina Editore.

PEnZIG Otto, 1924, Flora popolare italiana, Gênes, Orto Botanico della Regia Università.

PIERONI Andrea, 1999, « Botanica etnoveterinaria in Italia: lo stato attuale delle conoscenze », dans A. Pieroni (éd.), Herbs, Humans and Animals, Cologne, Experiences Verlag, p. 94-102.

PIGNOLI Maria Luisa, 2017, Les désignations des plantes sauvages dans les variétés arbëreshe (albanais d'Italie) : étude sémantique et motivationnelle, thèse de doctorat en cotutelle internationale, Université Côte d'Azur, Nice.

RESSULI Namik, 1986, Grammatica albanese, Bologne, Patron.

ReXhePi Besnik, Mustafa Behxhet, Hajdari Avni, Rushidi-ReXhePI Jehona, Quave Cassandra Leah \& PIERONI Andrea, 2013, « Traditional Medicinal Plant Knowledge among Albanians, Macedonians and Gorani in the Sharr Mountains (Republic of Macedonia) », Genet Resour Crop Evol, $\mathrm{n}^{\circ} 60$, p. 2055-2080.

RIEGLER Richard, 1981, « Lo zoomorfismo nelle tradizioni popolari », Quaderni di Semantica, vol. II, $\mathrm{n}^{\circ}$ 2, p. 305-324.

$\mathrm{SE}=$ ÇABEJ Eqrem, 1982-2014, Studime etimologjike në fushë të shqipes, Tirana, Akademia e Shkencave e Shqipërisë.

SKRED = MONIER-WILLIAMS Monier, 1960, A Sanskrit-English Dictionary, Oxford, University Press.

TRUMPER John Basset, 2007, « La structuration lexico-sémantique des phytonymes romans : le rôle du trait distinctif de la "rondeur" et la création de prototypes ", dans D. Trotter (éd.), Actes du $X X I V$ Congrès international de linguistique et de philologie romanes, Tübingen, Max Niemeyer, p. 241-258. 
TRUMPER John Basset, 2010, «Four Mainly Plant Squibs for a Well Known, Genial Squib-Writer », dans G. Belluscio et A. Mendicino (éds), Scritti in onore di Eric Pratt Hamp per il suo $90^{\circ}$ compleanno, Rende, Università della Calabria, p. 379-394.

UlLmann Stephen, 1975, Précis de sémantique française, Berne, Éditions A. Francke S.A.

Viegi Lucia, Pieroni Andrea, GuARrera Paolo Maria \& VANGelisti Roberta, 2003, « A Review of Plants Used in Folk Veterinary Medicine in Italy As Basis for a Databank », Journal of Ethnopharmacology, $\mathrm{n}^{\circ}$ 89, p. 221-244.

XHUVANI Aleksandër \& ÇABEJ Eqrem, 1962, «Prapashtesat e gjuhës shqipe », Studime gjuhësore, vol. 3, p. 191-300.

ZELENIN Dmitrij Konstantinovic, 1989, «Tabù linguistici nelle popolazioni dell'Europa orientale e dell'Asia settentrionale (II) », Quaderni di Semantica, vol. 10, n² 2, p. 123-180.

\section{ANNEXES}

\section{Abréviations}

\begin{tabular}{|l|l|}
\hline alb. & albanais \\
\hline all. & allemand \\
\hline all.dial. & allemand dialectal \\
\hline ar. & arabe \\
\hline arb. & arbëresh \\
\hline arm. & arménien \\
\hline aroum. & aroumain \\
\hline bos. & bosnien \\
\hline bulg. & bulgare \\
\hline cal. & calabrais \\
\hline eg. & égyptien \\
\hline g. & guègue \\
\hline gr.m. & grec moyen \\
\hline myc. & mycénien \\
\hline nap. & napolitain \\
\hline sic. & sicilien \\
\hline
\end{tabular}




\begin{tabular}{|l|l|}
\hline skr. & sanskrit \\
\hline slo. & slovène \\
\hline slvc. & slovaque \\
\hline t. & tosque \\
\hline tch. & tchèque \\
\hline tu. & turc \\
\hline v.h.a. & vieil haut allemand \\
\hline
\end{tabular}

\section{NOTES}

1. Nous utilisons le terme "phytomorphisme» pas dans le sens de représentation d'une plante par une autre plante, mais dans le sens que la désignation d'une plante est tirée d'une caractéristique de la plante elle-même.

\section{RÉSUMÉS}

Cette contribution fournit un aperçu de l'étude motivationnelle que nous avons conduite sur la phytonymie arbëreshe dans huit villages du sud de l'Italie où l'on parle des dialectes albanais. Cette recherche s'inscrit dans le contexte d'études concernant les ethnoclassements qui ont été lancées au moment où la géolinguistique a commencé à structurer sa contribution scientifique avec l'apport précieux fournit par les atlas linguistiques, notamment l'ALE et l'ALiR. Ces entreprises transnationales s'occupent d'étudier les faits lexicaux se basant sur le rôle fondamental que la motivation sémantique a pendant la création lexicale puisqu'elle représente un élément essentiel du processus de dénomination. L'analyse de la phytonymie arbëreshe permet, d'un côté, l'identification de quelques structures sémantiques communes sur lesquelles la création lexicale se base et, de l'autre côté, la connaissance approfondie de l'étymologie et, en diachronie, de la lexicologie albanaises.

This paper provides an overview of the motivational study carried out on the Arberresh phytonymy in eight villages in the south of Italy where Arbëreshë dialects are still spoken. This research is part of the general context concerning ethnoclassifications that started since geolinguistics began to structure its scientific contribution through linguistic atlases, in particular ALE and ALiR. These trans-national enterprises tackle the study of lexical facts considering as fundamental the role that semantic motivation plays during the lexical creation because it represents an essential component of the naming process. The Arberresh phytonymy analysis allows, on the one hand, identifying some common semantic patterns lexical creation is based on and, on the other hand, providing new insights into Albanian etymology and diachronic lexicology. 
INDEX

Mots-clés : phytonymie, dialectes albanais, motivation sémantique, géolinguistique

Keywords : plant names, Albanian dialects, semantic motivation, geolinguistics

\section{AUTEUR}

MARIA LUISA PIGNOLI

Université Côte d'Azur, BCL - Bases, Corpus, Langage (UMR 7320 - UNS/CNRS)

marialuisa.pignoli@gmail.com 